\title{
Rationale for the use of sphingosine analogues in COVID-19 patients
}

\author{
Authors: Deborah R Tasat ${ }^{A}$ and Juan S Yakisich ${ }^{B}$
}

\begin{abstract}
Despite the recent announcement of promising drug candidates to treat COVID-19, there is currently no effective antiviral drug or vaccine. There is strong evidence that acute lung injury/acute respiratory distress syndrome (ALI/ARDS), likely triggered by a cytokine storm, is responsible for the severity of disease seen in COVID-19 patients. In support of this hypothesis, pilot studies using IL-6 receptor inhibitors such as tocilizumab have shown promising results. Therefore, the use of drugs or cocktails of drugs with broader ability to inhibit these cytokine receptors is likely to be effective. In this article, we propose the use of sphingosine analogues, which have been shown to mitigate acute lung damage in animal models of ALI/ARDS, as early adjuvant therapies to prevent and/or mitigate the cytokine response in COVID-19 patients. This proposal is based on the ability of these drugs to decrease the production of IL- 6 and other cytokines. The potential application of fingolimod (FTY720), the oldest sphingosine analogue approved for the treatment of multiple sclerosis, in the early stages of COVID-19 is discussed in more detail as a prototype drug.
\end{abstract}

KEYWORDS: COVID-19, cytokines, cytokine storm, fingolimod, FTY720

DOI: 10.7861/clinmed.2020-0309

\section{Introduction}

The lack of specific treatment for patients with COVID-19 infection has triggered the search for treatment modalities including adjuvant drugs. Several existing drugs that have been approved for other diseases are being evaluated in pilot clinical trials in COVID-19 patients. The selection of these drugs is based on their effect on targets thought to be implicated in the pathophysiological mechanisms underpinning COVID-19 complications.

A significant number of patients have died from complications that at present are not clearly understood, but there is strong evidence that acute lung injury/acute respiratory distress syndrome (ALI/ARDS), likely triggered by a cytokine storm, may play a key role. ${ }^{2,3}$ Therefore, treatment for ALI/ARDS will play an

Authors: ${ }^{A}$ associate professor, National University of San Martin, San Martin, and University of Buenos Aires; ${ }^{B}$ assistant professor, Hampton University, Hampton, USA important role in decreasing the mortality of the disease. ALI/ ARDS are common conditions occurring in critically ill patients that are characterised by widespread pulmonary inflammation and by clinical symptoms such as hypoxemia, progressive dyspnea and even severe acute respiratory failure involving the lungs bilaterally. ${ }^{4}$ Cytokine storm is a systemic inflammatory response involving a plethora of cytokines. ${ }^{5}$ In COVID-19 patients the severity of the disease depends on the increase of pro-inflammatory factors such as IL-1, IL-2, IL-6, IL-7, IL-10, granulocyte-colony stimulating factor, interferon- $\boldsymbol{\gamma}$-inducible protein 10 , monocyte chemoattractant protein 1 , macrophage inflammatory protein-1 alpha, and TNF- $\alpha .{ }^{1}$ High levels of several cytokines (IL-2R, IL-6, IL-10, and TNF-a) were significantly associated with severe COVID-19 on admission. ${ }^{6}$ In addition, serum SARS-Cov-2 RNA (RNAaemia) was associated with elevated IL-6 concentration and poor prognosis in COVID-19 patients. ${ }^{7}$ Thus, blocking the biological activities of these cytokines, or at least the most important ones such as IL-6, may be beneficial for COVID-19 patients. This is the basis for the inclusion of tocilizumab (an IL- 6 receptor blocker) and other IL- 6 blockers in several treatment regimes. Tocilizumab appears to be an effective treatment option in COVID-19 patients at risk of cytokine storm as suggested by two pilot clinical studies ${ }^{8,9}$ and several case reports. ${ }^{10-15}$ It is natural to assume that to target the cytokine storm a cocktail of cytokine and/or cytokine receptor inhibitors is needed, which will result in a higher incidence of adverse effects. For instance, tocilizumab has been associated with hypertriglyceridemia in COVID-19 patients. ${ }^{16}$

Drugs with a broader ability to block cytokine activity may be more beneficial. In this article we propose the use of sphingosine analogues, in particular fingolimod, a drug approved for the treatment of relapsing-remitting (the most common) form of multiple sclerosis (MS). ${ }^{17,18}$ Fingolimod (FTY720, Gilenya ${ }^{\circledR}$ ) is a non-selective agonist of sphingosine 1-phosphate receptor (S1PR). It acts as a selective functional antagonist of the $\mathrm{S}_{1} \mathrm{P}_{1}$ subtype by inducing receptor downregulation. This downregulation leads to immunosuppression. ${ }^{19}$

Rationale for the use of fingolimod and other sphingosine analogues in COVID-19 patients

FTY720 and its derivative AAL-R are sphingosine analogues that are phosphorylated by sphingosine kinase 2 (SphK2), one of the enzymes responsible for the conversion of sphingosine into S1P. ${ }^{20}$ In an animal model of influenza, AAL-R significantly inhibited the production of IL-1 $\alpha$, IL-1 $\beta$, IL-6, IL-10, MCP-1, TNF- $\alpha$ and GM-CSF while maintaining protective neutralising antibody 
Host cells $\left(\mathrm{ACE} 2^{+}\right)$

Immune cells

Organ damage

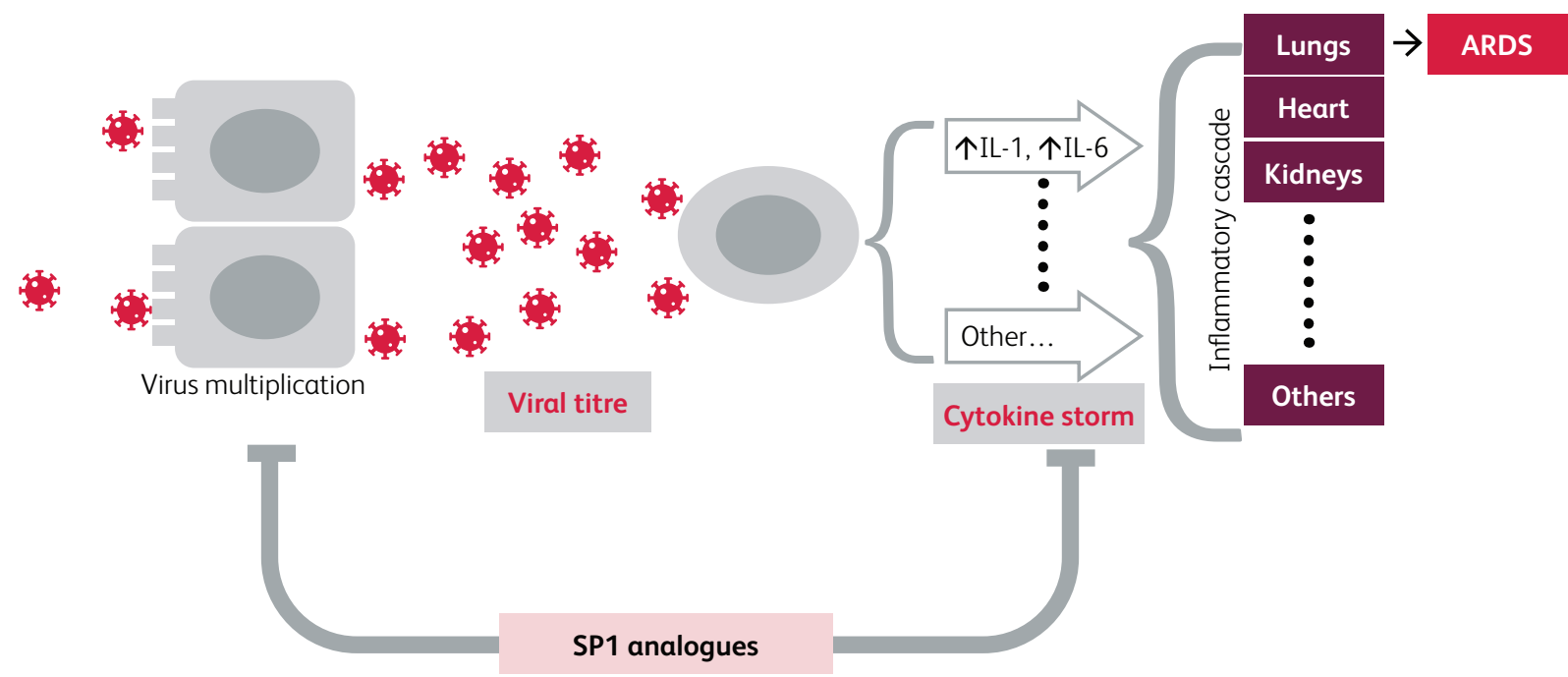

Fig 1. Mechanism of SARS-CoV-2-associated cytokine storm and associated cytokine storm-induced damage. Infection with SARS-CoV-2 in host $\left(A C E 2^{+}\right.$cells) cells followed by replication leads to high virus titre and stimulation of immune cells (eg macrophages, neutrophils, basophils) to produce and release high levels of cytokines ('cytokine storm'). The cytokine storm causes an adverse inflammatory cascade that damage several organs. Damage to the lungs leads to acute respiratory distress syndrome (ARDS). SP1 analogs have the potential to inhibit virus multiplication as well as to decrease the intensity of the cytokine storm. ${ }^{21,36}$

and cytotoxic T cell responses..$^{21}$ FTY720 alleviated podocyte injury in a rat model chronic kidney disease via downregulation of inflammatory cytokines including TNF- $\alpha$ and IL-6.22 In addition, FTY720 has protective effect on several other conditions such as stroke, probably by decreasing pro-inflammatory cytokines such high mobility group box 1 (HMGB1) and tumour necrosis factor- $\alpha$ $(\mathrm{TNF}-\alpha) .{ }^{23}$ Vascular endothelial cell (EC) barrier disruption may play a key role in ARDS; FTY720 analogues have been shown to potently enhance lung EC barrier function via $\mathrm{G}$ coupled receptor signaling in vitro ${ }^{24}$ and FTY720 has been shown to alleviate/ mitigate lung injuries in mice experimental models. ${ }^{25}$

There is therefore strong evidence that FTY720 has some type of 'pancytokine' or at least 'polycytokine' inhibiting properties and may therefore show more beneficial effects compared to selective cytokine inhibitors. At the same time, a polycytokine/pancytokine inhibitor may have more adverse effects. COVID-19 being a relatively new disease, information regarding the risk/benefit of targeting multiple cytokines is lacking. To our knowledge, there is only one ongoing clinical trial (at the recruiting stage) that proposed the use of fingolimod in patients with new coronavirus pneumonia (www.clinicaltrials.gov; NCT04280588). It is important to mention that in this clinical study the primary outcome measured is the change of the pneumonia severity after 5 days of treatment. Thus, the aim of this clinical trial is not to evaluate the preventive property of fingolimod on disease progression but its ability to change the severity once the pneumonia is already present.

\section{Rationale for the use of sphingosine analogues as early adjuvant therapy}

There are several reasons why it may be beneficial to use FTY720 at an earlier stage of infection to prevent or mitigate the severity of ALI/ARDS. First, while it is not known exactly when the cytokine storm starts in COVID-19 patients, cytokine levels at the time of admission to hospital are already several times higher than normal ${ }^{6}$ and thus preventing the rise of these cytokines at earlier stages may hold the key to mitigating the severity of the disease and decreasing hospital admissions. Second, infectious complications are relatively low in patients receiving sphingosine modulators. ${ }^{26}$ FTY720 has been prescribed for patients with relapsing-remitting multiple sclerosis to prevent the acute attacks since 2010 with good tolerability. ${ }^{17}$ Remarkably, the incidence of adverse events in patients treated with FTY720 for 12 and 24 months (the duration of treatment ranges from 6 to 24 months) was $7 \%$ and $14.8 \%$ and no death was reported even after 24 months of treatment. ${ }^{17}$ It is important to mention that when compared to placebo the incidence of adverse effects, while statistically significant, is not strikingly different (fingolimod $14.8 \%$ vs placebo $12.7 \%$ ). ${ }^{17}$ This observation suggests that FTY720 when administered for shorter periods (2-3 weeks) as we propose will be relatively safe.

Case studies reporting outcomes in COVID-19 patients who were already being treated with fingolimod for their MS using provide another rationale for the use of sphingosine analogues as early adjuvant therapy. Barzegar et al reported the positive outcome of a 42-year-old woman infected with COVID-19 with multiple comorbidities including multiple sclerosis, which was being treated with fingolimod. ${ }^{27}$ Malluci et a ${ }^{28}$ reported two cases of MS patients on fingolimod treatment who tested positive for COVID-19 but did not develop any symptoms, signs, or complications. Similar benign ${ }^{29}$ or more severe symptomatic but non-fatal ${ }^{30-32}$ cases of COVID-19 were reported in MS patients taking fingolimod. Conversely, an exacerbation of COVID-19 symptoms was observed when fingolimod was discontinued in one case report. ${ }^{33}$ A severe COVID-19 infection in a MS patient treated in whom fingolimod 
was discontinued was also reported; it is not clear when fingolimod was stopped ${ }^{34}$ but it is likely that the drugs were discontinued upon hospital admission due to concern that immunosuppression may worsen COVID-19.32

In addition, there are other novel sphingosine analogues available to treat MS such as siponimod (Mayzent), ozanimod (Zeposia) and ponesimod (not yet approved) that can be considered as alternatives. It was recently suggested that immunosuppression in MS may play a protective role in these patients. ${ }^{35}$ If this suggestion is proven in larger studies, other drugs used for the treatment of MS should be evaluated for their potential protective/preventive role in the progression of COVID-19 disease. Finally, recent in vitro data (from an article still in the preprint stage) demonstrated that nontoxic concentrations of fingolimod produced a 38.4-fold decrease of SARS-CoV-2 viral titer as compared to the DMSO control. ${ }^{36}$

\section{Conclusions}

The rationale to consider sphingosine analogues currently approved for the treatment of MS has been recently suggested by others. ${ }^{37}$ It use as early adjuvant therapy is supported by strong experimental data in other conditions relevant to COVID-19 disease as well as by recent several case reports with positive outcomes in COVID-19 patients taking fingolimod for MS treatment. Sphingosine analogues are relatively safe and well tolerated even when administered for prolonged times.

\section{References}

1 Zhang J, Xie B, Hashimoto K. Current status of potential therapeutic candidates for the COVID-19 crisis. Brain Behav Immun 2020;87:59-73.

2 Conti P, Ronconi G, Caraffa A et al. Induction of pro-inflammatory cytokines (IL-1 and IL-6) and lung inflammation by coronavirus-19 (COVID-19 or SARS-CoV-2): anti-inflammatory strategies. J Biol Regul Homeost Agents 2020;34:327-331.

3 Ye Q, Wang B, Mao J. The pathogenesis and treatment of the 'cytokine storm' in COVID-19. J Infect 2020;80:607-13.

4 Matthay MA, Zemans RL. The acute respiratory distress syndrome: pathogenesis and treatment. Annu Rev Pathol 2011;6:147-63.

5 Behrens EM, Koretzky GA. Review: cytokine storm syndrome: looking toward the precision medicine era. Arthritis Rheumatol 2017:69:1135-43

$6 \mathrm{Li} \mathrm{X,} \mathrm{Xu} \mathrm{S,} \mathrm{Yu} \mathrm{M} \mathrm{et} \mathrm{al.} \mathrm{Risk} \mathrm{factors} \mathrm{for} \mathrm{severity} \mathrm{and} \mathrm{mortality} \mathrm{in} \mathrm{adult}$ COVID-19 inpatients in Wuhan. J Allergy Clin Immunol 2020;146: $110-18$.

7 Chen X, Zhao B, Qu Y et al. Detectable serum SARS-CoV-2 viral load (RNAaemia) is closely correlated with drastically elevated interleukin 6 (IL-6) level in critically ill COVID-19 patients. Clin Infect Dis 2020, in press (doi: 10.1093/cid/ciaa449).

8 Jacobs JP, Stammers AH, St Louis ] et al. Extracorporeal membrane oxygenation in the treatment of severe pulmonary and cardiac compromise in COVID-19: Experience with 32 patients. ASAIO J 2020:66:722-30

9 Luo P, Liu Y, Qiu L et al. Tocilizumab treatment in COVID-19: A single center experience. J Med Virol 2020;92:814-18.

10 Cellina M, Orsi M, Bombaci F et al. Favorable changes of CT findings in a patient with COVID-19 pneumonia after treatment with tocilizumab. Diagn Interv Imaging 2020;101:323-4.

11 De Luna G, Habibi A, Deux JF et al. Rapid and severe Covid-19 pneumonia with severe acute chest syndrome in a sickle cell patient successfully treated with tocilizumab. Am J Hematol 2020;95:876-8.
12 Fontana F, Alfano G, Mori G et al. Covid-19 pneumonia in a kidney transplant recipient successfully treated with tocilizumab and hydroxychloroquine. Am J Transplant 2020;20:1902-6.

13 Michot JM, Albiges L, Chaput N et al. Tocilizumab, an anti-IL6 receptor antibody, to treat Covid-19-related respiratory failure: a case report. Ann Oncol 2020;31:961-4.

14 Mihai C, Dobrota R, Schroder M et al. COVID-19 in a patient with systemic sclerosis treated with tocilizumab for SSc-ILD. Ann Rheum Dis 2020;79:668-9.

15 Zhang X, Song K, Tong F et al. First case of COVID-19 in a patient with multiple myeloma successfully treated with tocilizumab. Blood Adv 2020;4:1307-10.

16 Morrison AR, Johnson JM, Ramesh M et al. Letter to the editor: Acute hypertriglyceridemia in patients with COVID-19 receiving tocilizumab. J Med Virol 2020, in press (doi:10.1002/jmv.25907)

17 La Mantia L, Tramacere I, Firwana B et al. Fingolimod for relapsingremitting multiple sclerosis. Cochrane Database Syst Rev 2016;4: CD009371.

18 Ziemssen T, Medin J, Couto CA, Mitchell CR. Multiple sclerosis in the real world: A systematic review of fingolimod as a case study. Autoimmun Rev 2017:16:355-76.

19 Huwiler A, Zangemeister-Wittke U. The sphingosine 1-phosphate receptor modulator fingolimod as a therapeutic agent: Recent findings and new perspectives. Pharmacol Ther 2018;185:34-49.

20 Seo Y], Pritzl C], Vijayan M et al. Sphingosine analogue AAL-R increases TLR7-mediated dendritic cell responses via p38 and type I IFN signaling pathways. J Immunol 2012;188:4759-68.

21 Marsolais D, Hahm B, Walsh KB et al. A critical role for the sphingosine analog AAL-R in dampening the cytokine response during influenza virus infection. Proc Natl Acad Sci U S A 2009;106:1560-5.

22 Su K, Zeng P, Liang W et al. FTY720 attenuates angiotensin II-induced podocyte damage via inhibiting inflammatory cytokines. Mediators Inflamm 2017;2017:3701385.

23 Dong YF, Guo RB, Ji ] et al. S1PR3 is essential for phosphorylated fingolimod to protect astrocytes against oxygen-glucose deprivationinduced neuroinflammation via inhibiting TLR2/4-NFKB signalling. J Cell Mol Med 2018;22:3159-66.

24 Wang L, Sammani S, Moreno-Vinasco L et al. FTY720 (s)-phosphonate preserves sphingosine 1-phosphate receptor 1 expression and exhibits superior barrier protection to FTY720 in acute lung injury. Crit Care Med 2014:42:e189-99.

25 Huang Z, Liu H, Zhang X et al. Transcriptomic analysis of lung tissues after hUC-MSCs and FTY720 treatment of lipopolysaccharide-induced acute lung injury in mouse models. Int Immunopharmacol 2018;63:26-34.

26 Giovannoni G, Hawkes C, Lechner-Scott ] et al. The COVID-19 pandemic and the use of MS disease-modifying therapies. Mult Scler Relat Disord 2020;39:102073.

27 Barzegar M, Mirmosayyeb O, Nehzat N et al. COVID-19 infection in a patient with multiple sclerosis treated with fingolimod. Neurol Neuroimmunol Neuroinflamm 2020;7:e753.

28 Mallucci G, Zito A, Fabbro BD, Bergamaschi R. Asymptomatic SARS-CoV-2 infection in two patients with multiple sclerosis treated with fingolimod. Mult Scler Relat Disord 2020:45:102414.

29 Bollo L, Guerra T, Bavaro DF et al. Seroconversion and indolent course of COVID-19 in patients with multiple sclerosis treated with fingolimod and teriflunomide. J Neurol Sci 2020;416:117011.

30 Arca KN, Starling AJ. Treatment-refractory headache in the setting of COVID-19 pneumonia: migraine or meningoencephalitis? SN Compr Clin Med 2020, in press (doi: 10.1007/s42399-02000369-y).

31 Chiarini M, Paghera S, Moratto D et al. Immunologic characterization of a immunosuppressed multiple sclerosis patient that recovered from SARS-CoV-2 infection. J Neuroimmunol 2020;345:577282. 
32 Valencia-Sanchez C, Wingerchuk DM. A fine balance: Immunosuppression and immunotherapy in a patient with multiple sclerosis and COVID-19. Mult Scler Relat Disord 2020;42:102182.

33 Gomez-Mayordomo V, Montero-Escribano P, Matías-Guiu JA et al. Clinical exacerbation of SARS-CoV2 infection after fingolimod withdrawal. J Med Virol 2020, in press (doi: 10.1002/jmv.26279).

34 Foerch C, Friedauer L, Bauer B et al. Severe COVID-19 infection in a patient with multiple sclerosis treated with fingolimod. Mult Scler Relat Disord 2020;42:102180.

35 Novi G, Mikulska M, Briano F et al. COVID-19 in a MS patient treated with ocrelizumab: does immunosuppression have a protective role? Mult Scler Relat Disord 2020:42:102120.
36 Risner KH, Tieu KV, Wang Y et al. Maraviroc inhibits SARS-CoV-2 multiplication and s-protein mediated cell fusion in cell culture. bioRxiv 2020; 2020.08.12.246389.

37 Naz F, Arish M. Battling COVID-19 pandemic: sphingosine-1Phosphate analogs as an adjunctive therapy? Front Immunol 2020;11:1102.

Address for correspondence: Dr Juan S Yakisich, 200 William R Harvey Way, Department of Pharmaceutical Sciences, Hampton University, Hampton, VA 23668, USA.

Email: juan.yakisich@hamptonu.edu 